\title{
Clinically Oriented Research in Transfusion Medicine
}

\author{
Petra Krakowitzky Walter Sibrowski \\ Institut für Transfusionsmedizin, Universitätsklinikum Münster, Deutschland
}

The health state of the donor primarily affects the quality and safety of blood products. Therefore, the donor questionnaire, the suitability examination of the donor, and the medical approval for donation are of utmost importance for the high quality of blood products. In addition to known infectiological and immunological risks, medicines taken by the donor before blood donation are associated with hardly calculable risks for the blood acceptor. Therefore, donors under medication normally are not accepted for donation. Donors frequently ingest drugs like ASS to conquer lighter disturbances of health without being aware that this is also a medical treatment. It is well known that ASS irreversibly inhibits platelet function, resulting in a therapeutic ineffectiveness of a blood product manufactured from such a donation. Besides pharmaceutical drugs, also alcohol or, with reservations, tobacco intake by the donor may affect the quality of the blood product. The threat of the patient by such blood products has to be taken serious even if the effects of such legal drugs on blood product quality, except for alcohol, are not investigated in detail.

In their article Becker et al. [1] present an interesting concept how the rate of donors being rejected because of drug intake before donation can be reduced. They investigated red cell concentrates of probands with medication intake before donation and determined the remaining concentration of the respective drug in the blood product. Nowadays blood donation services are confronted with the problem of medication intake in daily routine, and especially older donors must be deferred from donation temporarily or permanently. Simpler decision criteria and new findings concerning the remaining drug concentrations my contribute to an easier approval of urgently needed blood donations. Whether the provocative conclusions of Becker et al. [1] will find high acceptance by transfusion specialists, is still not foreseeable.

Reichelt et al. [2] describe the effects of age, sex, and smoker status on mobilization of CD34+/CD133+ stem cells.
CD133 antigen was first described 10 years ago and is co-expressed on approximately $75 \%$ of CD34+ hematopoietic stem cells, especially on CD34 bright cells. Meanwhile an immunomagnetic purification technique for this antigen is available for clinical use. As shown by Reichelt et al. [2], mobilization of these cells significantly differs only between sexes. Apparently in male donors more stem cells can be mobilized than in female donors. What caused these sex specific differences in stem cell mobilization is not known thus far.

Two further original articles in this issue highlight the quality of the blood products plasma and platelets. Gravemann et al. [3] examined the thrombin formation rate of plasma pathogen-inactivated by methylene blue / light treatment. Picker et al. [4] describe the storage-dependent changes of pathogen-inactivated platelet concentrates over 7 days after Mirasol-PRT treatment. In this respect it is of particular interest that the release of inflammatory cytokines is increased after MirasolPRT treatment, probably resulting in a higher rate of febrile transfusion reactions in recipients of such blood products.

Three case reports [5-7] complete this overview on current clinical transfusion medicine.

The current issue of Transfusion Medicine and HemoTHERAPY gives a comprehensive insight in modern transfusion medicine and its various clinically oriented research topics. As it does not deal with popular and currently questioned topics, such clinically focused research nowadays is in little demand. Thus, there is great difficulty to receive third-party and university funds for such a research. However, even this research is indispensable for a broadly based and sustainable patient care. The many small advances in quality and safety of blood products for our patients that were made possible by the multitude of such research projects as well as the research and development work of many blood donation services has substantially contributed to implement a high level of health protection in Germany. Keeping this in mind, it does not seem absurd to

\section{KARGER}

Fax +497614520714

Information@Karger.de

www.karger.com (c) 2009 S. Karger GmbH, Freiburg

Accessible online at:

www.karger.com/tmh
Prof. Dr. med. Dr. rer. nat. Walter Sibrowski

Institut für Transfusionsmedizin

Universitätsklinikum Münster

Domagkstraße 11, 48149 Münster, Germany

Tel. $+4925183557-68$, Fax -62

E-mail sibrowski@uni-muenster.de 
propose that also in the future there will be a great demand for such clinically oriented research and development. The unspectacular results of this research may help many patients in overcoming or palliating their frequently serious diseases. However, external financing of such patient-oriented research in the field of save blood supply may become more and more impossible in the current funding systems of Germany and the EU. Nevertheless, it is of utmost importance for the specialty of transfusion medicine to support, encourage and advance the product development in the field of classic blood components and innovative cellular therapeutics by their own and thus to further contribute to an improvement of patient care.

\section{References}

1 Becker CDK, Stichtennoth D, Wichmann MG, Schaefer C, Szinicz L: Blood donors on medication - an approach to minimize drug burden for recipients of blood products and to limit deferral of donors. Transfus Med Hemother 2009;36(2):107-113.

2 Reichelt H, Barz D, Thude H: CD34+ and CD133+ primitive stem cell expression in peripheral blood: considering gender, age, and smoking. Transfus Med Hemother 2009;36(2):129-134.

3 Gravemann U, Kusch N, Koenig H, Mohr H, Müller TH: Thrombin generation capacity of methylene blue-treated plasma prepared by the Theraflex MB Plasma System. Transfus Med Hemother 2009;36(2):122-127.
Picker SM, Steisel A, Gathof BS: Evaluation of white-blood-cell and platelet-derived cytokine accumulation in Mirasol-PRT treated platelets. Transfus Med Hemother 2009;36(2):114-120.

5 Kerz T, Schinzel, H: Anticoagulation in a neurosurgical patient with heparin-induced thrombocytopenia (HIT type II) with argatroban and fondaparinux after clipping of an intracranial aneurysm. Transfus Med Hemother 2009;36(2):141-143.
6 Hacioglu S, Altuntas F, Kaynar L, Sari I, Patiroglu $\mathrm{T}$, Cetin M: Rhabdomyolysis in a healthy peripheral blood stem cell donor following mobilization with filgrastim. Transfus Med Hemother 2009; 36(2):135-137.

7 Mansouritorghabeh H, Rezaieyazdi Z, Bagheri M: Successful use of factor VIII concentrate and fresh frozen plasma for four dental extractions in an individual with combined factor V and VIII deficiency. Transfus Med Hemother 2009;36(2):138-139. 